\title{
MHD FLOW PAST A PARABOLIC FLOW PAST AN INFINITE ISOTHERMAL VERTICAL PLATE IN THE PRESENCE OF THERMAL RADIATION AND CHEMICAL REACTION
}

\author{
R. MUTHUCUMARASWAMY* \\ Department of Applied Mathematics \\ Sri Venkateswara College of Engineering \\ Irungattukottai, Sriperumbudur-602117, INDIA \\ E-mail: msamy@svce.ac.in \\ P. SIVAKUMAR \\ Department of Mathematics \\ P.B.College of Engineering \\ Irungattukottai, Sriperumbudur, Chennai-602117, INDIA
}

\begin{abstract}
The problem of MHD free convection flow with a parabolic starting motion of an infinite isothermal vertical plate in the presence of thermal radiation and chemical reaction has been examined in detail in this paper. The fluid considered here is a gray, absorbing emitting radiation but a non-scattering medium. The dimensionless governing coupled linear partial differential equations are solved using the Laplace transform technique. A parametric study is performed to illustrate the influence of the radiation parameter, magnetic parameter, chemical reaction parameter, thermal Grashof number, mass Grashof number, Schmidt number and time on the velocity, temperature, concentration. The results are discussed graphically and qualitatively. The numerical results reveal that the radiation induces a rise in both the velocity and temperature, and a decrease in the concentration. The model finds applications in solar energy collection systems, geophysics and astrophysics, aerospace and also in the design of high temperature chemical process systems.
\end{abstract}

Key words: MHD, isothermal, radiation, vertical plate, thermal radiation, chemical reaction, Laplace transforms technique.

\section{Introduction}

In nature, there exist flows which are caused not only by the temperature differences but also the concentration differences. These mass transfer differences do affect the rate of heat transfer. In industries, many transport processes exist in which heat and mass transfer takes place simultaneously as a result of combined buoyancy effect in the presence of thermal radiation. Hence, radiative heat and mass transfer play an important role in manufacturing industries for the design of fins, steel rolling, nuclear power plants, gas turbines and various propulsion devices for aircraft, missiles, satellites, combustion and furnace design, materials processing, energy utilization, temperature measurements, remote sensing for astronomy and space exploration, food processing and cryogenic engineering, as well as numerous agricultural, health and military applications. If the temperature of the surrounding fluid is rather high, radiation effects play an important role and this situation does exist in space technology.

The study of magneto hydro-dynamics with mass and heat transfer in the presence of radiation and diffusion has attracted the attention of a large number of scholars due to diverse applications. In astrophysics and geophysics, it is applied to study the stellar and solar structures, radio propagation through the

\footnotetext{
* To whom correspondence should be addressed
} 
ionosphere, etc. In engineering we find its applications in MHD pumps, MHD bearings, etc. The phenomenon of mass transfer is also very common in the theory of stellar structure and observable effects are detectable on the solar surface. In power engineering, the thermal physics of hydro magnetic problems with mass transfer have enormous applications. Radiative flows are encountered in many industrial and environment processes, e.g., heating and cooling chambers, fossil fuel combustion energy processes, evaporation from large open water reservoirs, astrophysical flows, and solar power technology and space vehicle re-entry. On the other hand, hydro magnetic free convective flows with heat and mass transfer through a porous medium have many important applications such as oil and gas production, geothermal energy, cereal grain storage, in chemical engineering for filtration and purification process, in agriculture engineering to study the underground water resources and porous insulation. In view of these applications, the unsteady magneto hydrodynamic incompressible viscous flows past an infinite vertical plate thorough a porous medium have received much attention.

The study of heat and mass transfer problems with chemical reaction is of great practical importance to engineers and scientists because of its almost universal occurrence in many branches of science and engineering. A few representative fields of interest in which combined heat and mass transfer along with chemical reaction play an important role are found in chemical process industries such as food processing and polymer production. Chambre and Young [1] analyzed a first-order chemical reaction in the neighborhood of a horizontal plate. Das et al. [2] studied the effect of a homogeneous first-order chemical reaction on the flow past an impulsively started infinite vertical plate with uniform heat flux and mass transfer. Again, mass transfer effects on a moving isothermal vertical plate in the presence of chemical reaction was studied by Das et al. [3]. Muthucumaraswamy and Meenakshisundaram [4] studied chemical reaction effects on a vertical oscillating plate with variable temperature. Their dimensionless governing equations were solved by the usual Laplace transform technique.

Gupta et al. [5] studied free convection effects on flow past a linearly accelerated vertical plate in the presence of viscous dissipative heat using the perturbation method. Kafousias and Raptis [6] extended this problem to include mass transfer effects subjected to variable suction or injection. Free convection effects on flow past an exponentially accelerated vertical plate was studied by Singh and Kumar [7]. The skin friction for an accelerated vertical plate was studied analytically by Hossain and Shayo [8]. Jha et al. [9] analyzed mass transfer effects on an exponentially accelerated infinite vertical plate with constant heat flux and uniform mass diffusion. Recently, Muthucumaraswamy et al. [10] studied mass transfer effects on an exponentially accelerated isothermal vertical plate. Soundalgekar and Takhar [11] considered the radiative free convective flow of an optically thin gray-gas past a semi-infinite vertical plate. Radiation effects on mixed convection along an isothermal vertical plate were studied by Hossain and Takhar [12]. The governing equations were solved analytically. Das et al. [13] analyzed radiation effects on flow past an impulsively started infinite isothermal vertical plate. The governing equations were solved by the Laplace transform technique. Muthucumaraswamy and Janakiraman [14] studied MHD and radiation effects on a moving isothermal vertical plate with variable mass diffusion.

It is proposed to study the effects of MHD flow with a parabolic starting motion of a infinite isothermal vertical plate in the presence of thermal radiation and chemical reaction. The dimensionless governing equations are solved using the Laplace transform technique. The solutions are in terms of exponential and complementary error function

\section{Mathematical analysis}

The unsteady flow of a viscous incompressible fluid with a parabolic started motion of an infinite isothermal vertical plate in the presence of thermal radiation and chemical reaction of first order has been considered. The $x^{\prime}$-axis is taken along the plate in the vertically upward direction and the $y$-axis is taken normal to the plate. At time $t^{\prime} \leq 0$, the plate and fluid are at the same temperature $T_{\infty}$ and concentration $C_{\infty}^{\prime}$. At time $t^{\prime}>0$, the plate is started with a velocity $u=u_{0} t^{\prime 2}$ in its own plane against the gravitational field. 
The temperature from the plate is raised to $T_{w}$ and the concentration level near the plate are also raised to $C_{w}^{\prime}$. A chemically reactive species which transforms according to a simple reaction involving the concentration is emitted from the plate and diffuses into the fluid. The plate is also subjected to a uniform magnetic field of strength $B_{0}$ which is assumed to be applied normal to the plate. The reaction is assumed to take place entirely in the stream. Then under usual Boussinesq's approximation the unsteady parabolic starting motion is governed by the following equations

$$
\begin{aligned}
& \frac{\partial u}{\partial t^{\prime}}=g \beta\left(T-T_{\infty}\right)+g \beta *\left(C^{\prime}-C_{\infty}^{\prime}\right)+v \frac{\partial^{2} u}{\partial y^{2}}-\frac{\sigma B_{0}^{2}}{\rho} u, \\
& \rho C_{p} \frac{\partial T}{\partial t^{\prime}}=k \frac{\partial^{2} T}{\partial y^{2}}-\frac{\partial q_{r}}{\partial y}, \\
& \frac{\partial C^{\prime}}{\partial t^{\prime}}=D \frac{\partial^{2} C^{\prime}}{\partial y^{2}}-k_{l}\left(C^{\prime}-C_{\infty}^{\prime}\right) .
\end{aligned}
$$

With the following initial and boundary conditions

$$
\begin{array}{lll}
u=0, & T=T_{\infty}, & C^{\prime}=C_{\infty}^{\prime} \quad \text { for all } \quad y, t^{\prime} \leq 0, \\
t^{\prime}>0: & u=u_{0} \cdot t^{\prime 2}, \quad T=T_{w}, \quad C^{\prime}=C_{w}^{\prime} \quad \text { at } \quad y=0, \\
u \rightarrow 0 \quad T \rightarrow T_{\infty}, \quad C^{\prime} \rightarrow C_{\infty}^{\prime} \quad \text { as } \quad y \rightarrow \infty .
\end{array}
$$

The local radiant for the case of an optically thin gray gas is expressed by

$$
\frac{\partial q_{r}}{\partial y}=-4 a^{*} \sigma\left(T_{\infty}^{4}-T^{4}\right)
$$

It is assumed that the temperature differences within the flow are sufficiently small such that $T^{4}$ may be expressed as a linear function of the temperature. This is accomplished by expanding $T^{4}$ in a Taylor series about $T_{\infty}$ and neglecting higher-order terms, thus

$$
T^{4} \cong 4 T_{\infty}^{3} T-3 T_{\infty}^{4}
$$

By using Eqs (2.5) and (2.6), Eq.(2.2) reduces to

$$
\rho C_{p} \frac{\partial T}{\partial t^{\prime}}=k \frac{\partial^{2} T}{\partial y^{2}}+16 a^{*} \sigma T_{\infty}^{3}\left(T_{\infty}-T\right) .
$$

On introducing the following non-dimensional quantities 


$$
\begin{aligned}
& U=u\left(\frac{u_{0}}{v^{2}}\right)^{1 / 3}, \quad t=\left(\frac{u_{0}^{2}}{v}\right)^{1 / 3} t^{\prime}, \quad Y=y\left(\frac{u_{0}}{v^{2}}\right)^{1 / 3}, \\
& \theta=\frac{T-T_{\infty}}{T_{w}-T_{\infty}}, \quad C=\frac{C^{\prime}-C_{\infty}^{\prime}}{C_{w}^{\prime}-C_{\infty}^{\prime}}, \quad \mathrm{Gr}=\frac{g \beta\left(T_{w}-T_{\infty}\right)}{\left(v \cdot u_{0}\right)^{1 / 3}}, \quad \mathrm{Gc}=\frac{g \beta\left(C_{w}^{\prime}-C_{\infty}^{\prime}\right)}{\left(v \cdot u_{0}\right)^{1 / 3}}, \\
& R=\frac{16 a^{*} \sigma \cdot T_{\infty}^{3}}{k}\left(\frac{v^{2}}{u_{0}}\right)^{\frac{2}{3}}, \quad K=K_{l}\left(\frac{v}{u_{0}^{2}}\right)^{\frac{1}{3}}, \quad M=\frac{\sigma B_{0}^{2}}{\rho}\left(\frac{v}{u_{o}^{2}}\right)^{\frac{1}{3}}, \quad \operatorname{Pr}=\frac{\mu C_{p}}{k}, \quad \mathrm{Sc}=\frac{v}{D}
\end{aligned}
$$

Eqs (2.1), (2.3) and (2.7) reduce to the following dimensionless form

$$
\begin{aligned}
& \frac{\partial U}{\partial t}=\operatorname{Gr} \theta+\operatorname{GcC}+\frac{\partial^{2} U}{\partial Y^{2}}-M U, \\
& \frac{\partial \theta}{\partial t}=\frac{1}{\operatorname{Pr}} \frac{\partial^{2} \theta}{\partial Y^{2}}-\frac{R}{\operatorname{Pr}} \theta, \\
& \frac{\partial C}{\partial t}=\frac{1}{\operatorname{Sc}} \frac{\partial^{2} C}{\partial Y^{2}}-K C .
\end{aligned}
$$

The corresponding initial and boundary conditions in a dimensionless form are as follows

$$
\begin{aligned}
& U=0, \quad \theta=0, \quad C=0 \quad \text { for all } \quad Y, t \leq 0 \\
& t>0: \quad U=t^{2}, \quad \theta=1, \quad C=1 \quad \text { at } \quad Y=0, \\
& U \rightarrow 0, \quad \theta \rightarrow 0, \quad C \rightarrow 0 \quad \text { as } \quad Y \rightarrow \infty .
\end{aligned}
$$

The dimensionless governing Eqs (2.9) to (2.11) and the corresponding initial and boundary conditions (2.12) are tackled using the Laplace transform technique.

$$
\begin{aligned}
& \theta=\frac{1}{2}[\exp (2 \eta \sqrt{\operatorname{Pr} a t}) \operatorname{erfc}(\eta \sqrt{\operatorname{Pr}}+\sqrt{a t})+\exp (-2 \eta \sqrt{\operatorname{Pr} a t}) \operatorname{erfc}(\eta \sqrt{\operatorname{Pr}}-\sqrt{a t})], \\
& c=\frac{1}{2}[\exp (2 \eta \sqrt{\operatorname{Sc} K t}) \operatorname{erfc}(\eta \sqrt{\operatorname{Sc}}+\sqrt{K t})+\exp (-2 \eta \sqrt{\operatorname{Sc} K t}) \operatorname{erfc}(\eta \sqrt{\operatorname{Sc}}-\sqrt{K t})],
\end{aligned}
$$




$$
\begin{aligned}
& u=2\left(\frac{\left(\eta^{2}+M t\right) t}{4 M}[\exp (2 \eta \sqrt{M t}) \operatorname{erfc}(\eta+\sqrt{M t})+\exp (-2 \eta \sqrt{M t}) \operatorname{erfc}(\eta-\sqrt{M t})]+\right. \\
& +\frac{\eta \sqrt{t}(1-4 M t)}{8 M^{3 / 2}}[\exp (-2 \eta \sqrt{M t}) \operatorname{erfc}(\eta-\sqrt{M t})-\exp (2 \eta \sqrt{M t}) \operatorname{erfc}(\eta+\sqrt{M t})]+ \\
& -\frac{\eta t}{2 M \sqrt{\pi}} \exp \left(-\left(\eta^{2}+M t\right)\right)+d\left(\frac{1}{2}[\exp (2 \eta \sqrt{M t}) \operatorname{erfc}(\eta+\sqrt{M t})+\exp (-2 \eta \sqrt{M t}) \operatorname{erfc}(\eta-\sqrt{M t})]+\right. \\
& -\frac{\exp (b t)}{2}[\exp (2 \eta \sqrt{(M+b) t}) \operatorname{erfc}(\eta+\sqrt{(M+b) t})+\exp (-2 \eta \sqrt{(M+b) t}) \operatorname{erfc}(\eta-\sqrt{(M+b) t})]+ \\
& -\frac{1}{2}[\exp (2 \eta \sqrt{\operatorname{Pr} a t}) \operatorname{erfc}(\eta \sqrt{\operatorname{Pr}}+\sqrt{a t})+\exp (-2 \eta \sqrt{\operatorname{Pr} a t}) \operatorname{erfc}(\eta \sqrt{\operatorname{Pr}}-\sqrt{a t})]+ \\
& +\frac{\exp (b t)}{2}[\exp (2 \eta \sqrt{\operatorname{Pr}(a+b) t}) \operatorname{erfc}(\eta \sqrt{\operatorname{Pr}}+\sqrt{(a+b) t})+\exp (-2 \eta \sqrt{\operatorname{Pr}(a+b) t}) \operatorname{erfc}(\eta \sqrt{\operatorname{Pr}}-\sqrt{(a+b) t})]+ \\
& +e\left(\frac{1}{2}[\exp (2 \eta \sqrt{M t}) \operatorname{erfc}(\eta+\sqrt{M t})+\exp (-2 \eta \sqrt{M t}) \operatorname{erfc}(\eta-\sqrt{M t})]+\right. \\
& -\frac{\exp (c t)}{2}[\exp (2 \eta \sqrt{(M+c) t}) \operatorname{erfc}(\eta+\sqrt{(M+c) t})+\exp (-2 \eta \sqrt{(M+c) t}) \operatorname{erfc}(\eta-\sqrt{(M+c) t})]+ \\
& -\frac{1}{2}[\exp (2 \eta \sqrt{\operatorname{Sc} K t}) \operatorname{erfc}(\eta \sqrt{\operatorname{Sc}}+\sqrt{K t})+\exp (-2 \eta \sqrt{\operatorname{Sc} K t}) \operatorname{erfc}(\eta \sqrt{\operatorname{Sc}}-\sqrt{K t})]+ \\
& \left.+\frac{\exp (c t)}{2}[\exp (2 \eta \sqrt{\operatorname{Sc}(K+c) t}) \operatorname{erfc}(\eta \sqrt{\operatorname{Sc}}+\sqrt{(K+c) t})+\exp (-2 \eta \sqrt{\operatorname{Sc}(K+c) t}) \operatorname{erfc}(\eta \sqrt{\operatorname{Sc}}-\sqrt{(K+c) t})]\right)
\end{aligned}
$$

where $\quad a=\frac{R}{\operatorname{Pr}}, \quad b=\frac{R-M}{1-\operatorname{Pr}}, \quad c=\frac{\mathrm{Sc} K-M}{1-\mathrm{Sc}}, \quad d=\frac{\mathrm{Gr}}{b(1-\mathrm{Pr})}, \quad e=\frac{\mathrm{Gc}}{c(1-\mathrm{Sc})} \quad$ and $\quad \eta=\frac{y}{2 \sqrt{t}}$.

\section{Discussion of results}

To interpret the results for a better understanding of the problem, numerical computations are carried out for different physical parameters $K, R, M, t, \mathrm{Sc}$, and $\mathrm{Gr}, \mathrm{Gc}$, upon the nature of the flow and transport. The value of the Schmidt number Sc is taken to be 0.6 which corresponds to water-vapor. The value of the Prandtl number $\mathrm{Pr}$ is chosen such that it represents air $(\mathrm{Pr}=0.71)$. The numerical values of the velocity, temperature and concentration are computed for the above mentioned parameters.

The concentration profiles for different values of the chemical reaction parameter $(K=0.2,2,5$, $10), \mathrm{Sc}=0.6$ and $t=0.2$ are shown in Fig. 1 . The effect of the chemical reaction parameter plays an important role in the concentration field. It is observed that the plate concentration decreases with increasing values of the chemical reaction parameter. The velocity profiles for different values of the chemical reaction parameter $(K=2,5,10), \mathrm{Gr}=2, \mathrm{Gc}=5, \operatorname{Pr}=0.71$ and $t=0.2$ are shown in Fig.2.

Figure 3 illustrates the effects of the magnetic field parameter on velocity when $(M=2,4,7)$, $R=K=10, \mathrm{Gr}=2, \mathrm{Gc}=5$ and $t=0.4$. It is observed that velocity increases with decreasing values of the magnetic field parameter. This shows that the increase in the magnetic field parameter leads to a fall in velocity. This agrees with the expectations, since the magnetic field exerts a retarding force on the free convective flow.

Figure 4 demonstrates the effects of the radiation parameter on velocity when $(R=5,10,15), M=$ $2, K=2, \mathrm{Gr}=\mathrm{Gc}=5$ and $t=0.2$. It is observed that velocity increases with the decreasing thermal radiation parameter. The trend shows that velocity is suppressed due to higher thermal radiation. 


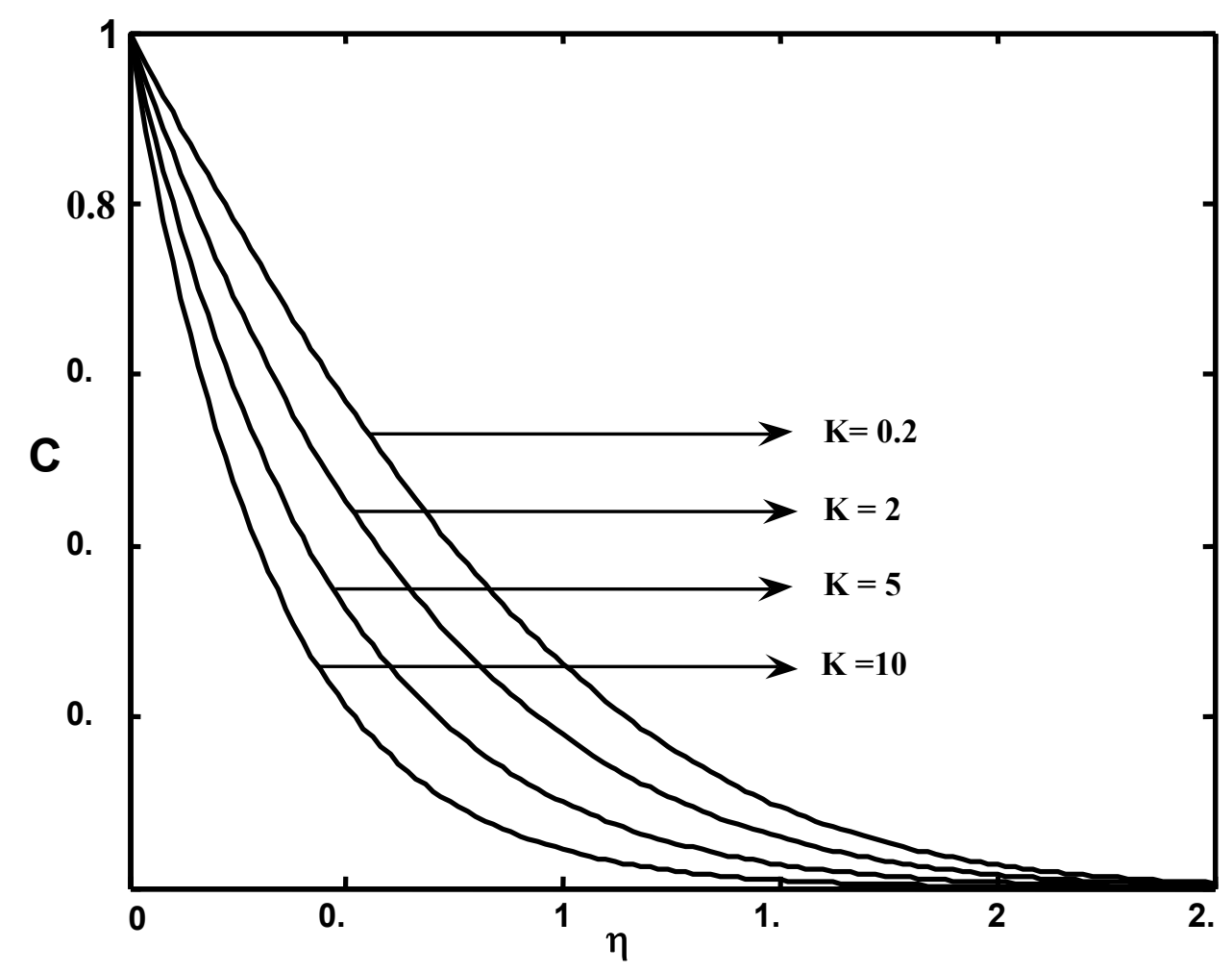

Fig.1. Concentration profiles for different values of $K$.

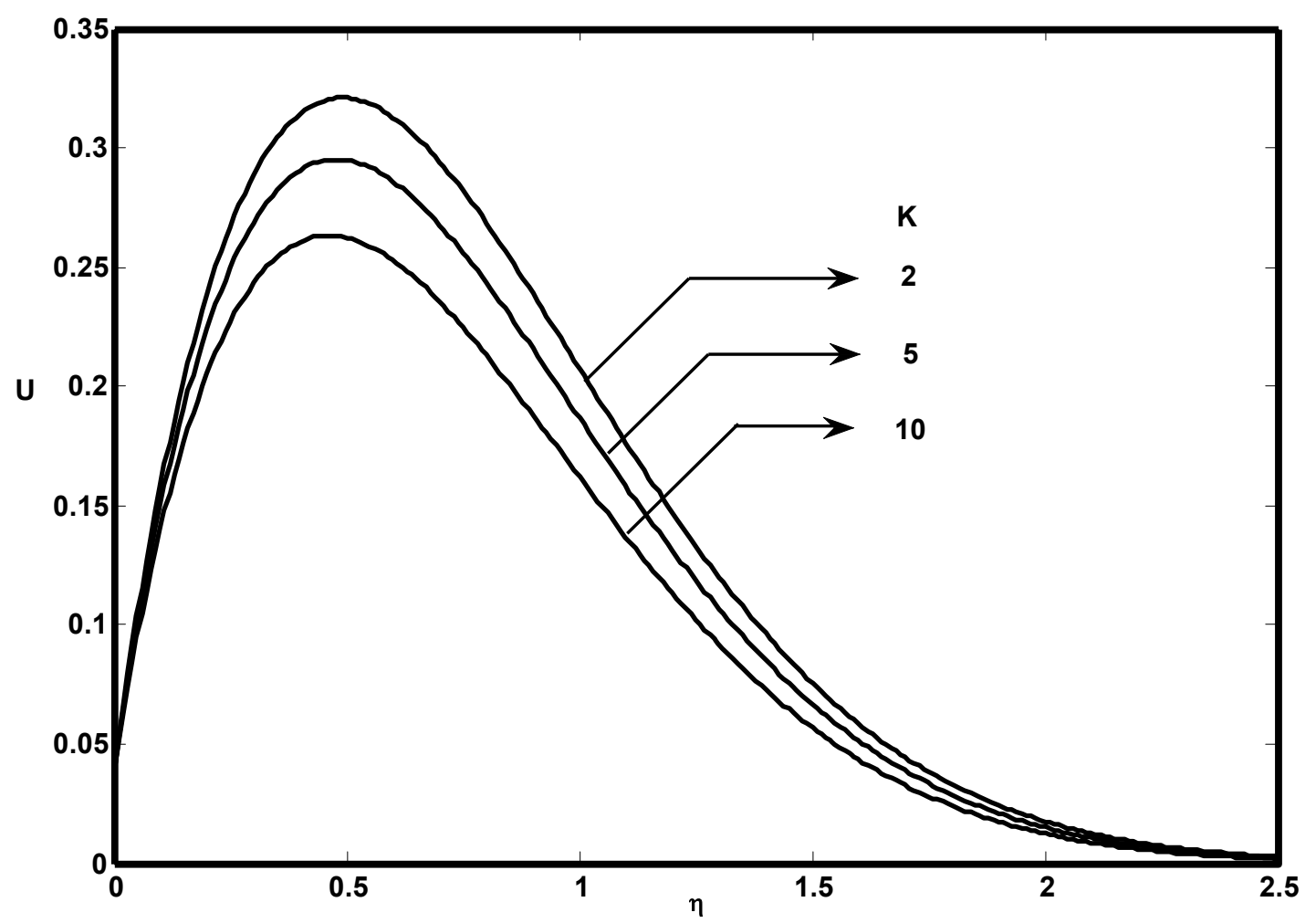

Fig.2. Velocity profiles for different values of $K$. 


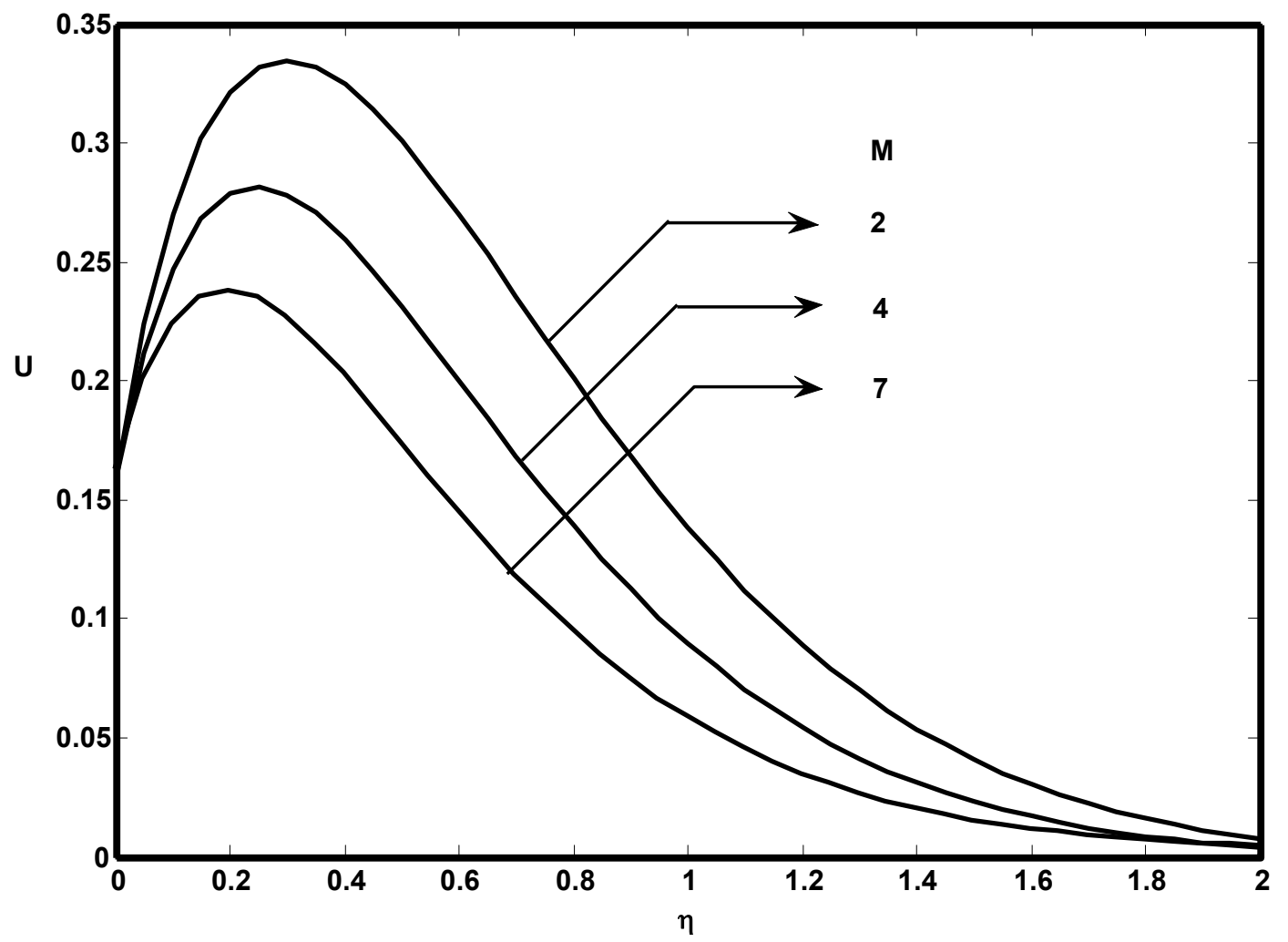

Fig.3. Velocity profiles for different values of $M$.

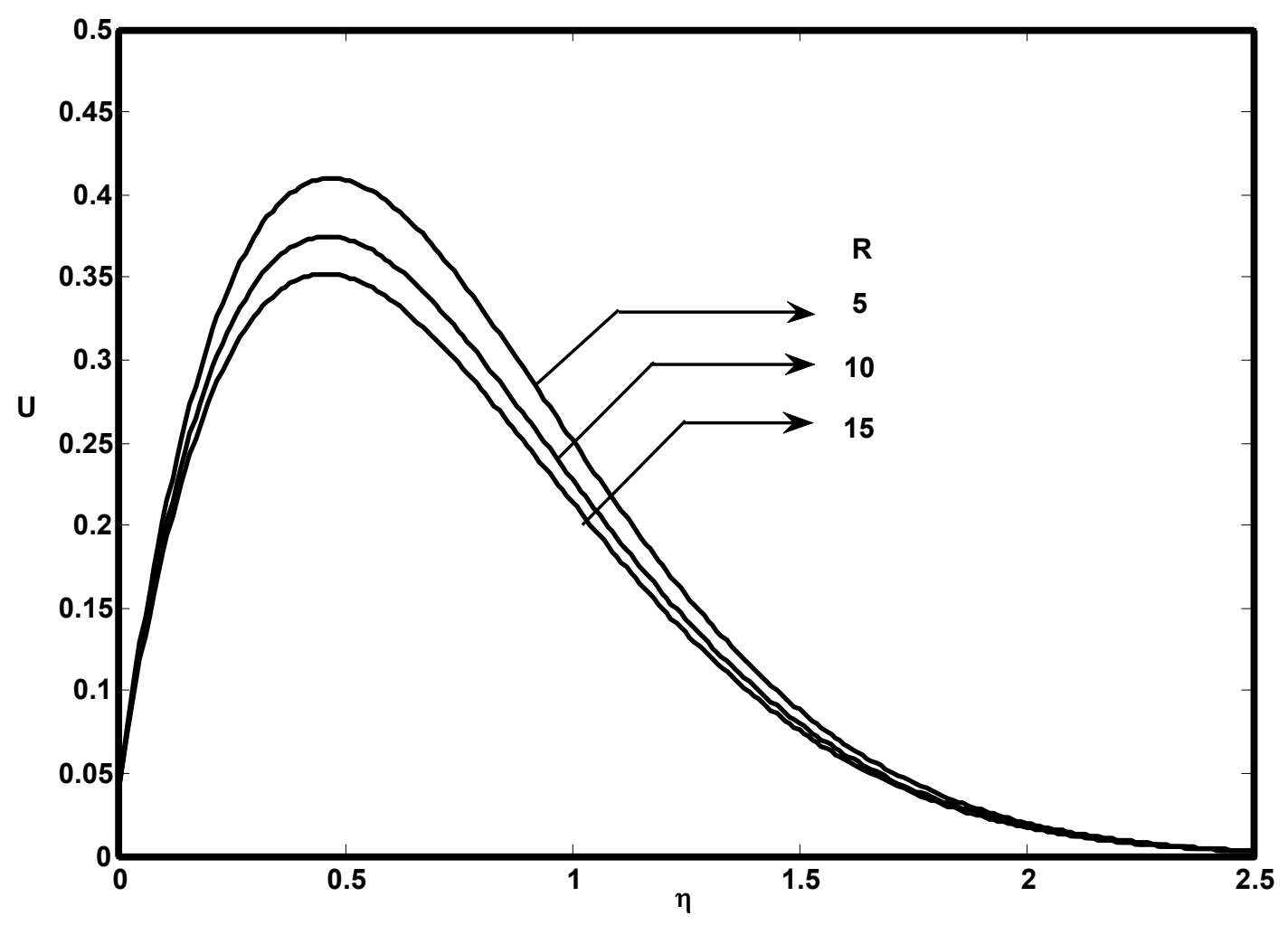

Fig.4. Velocity profiles for different values of $R$. 


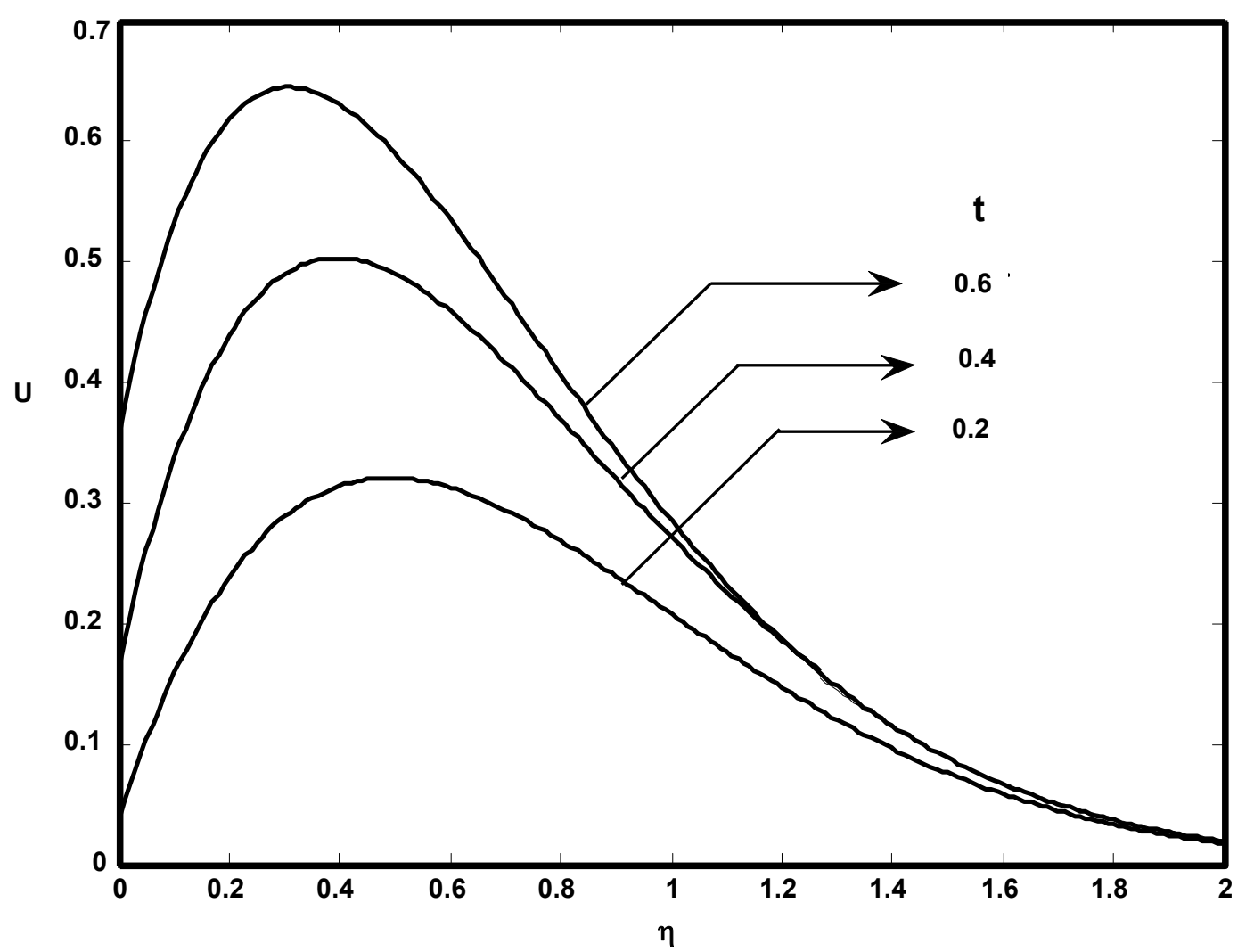

Fig.5. Velocity profiles for different values of $t$.

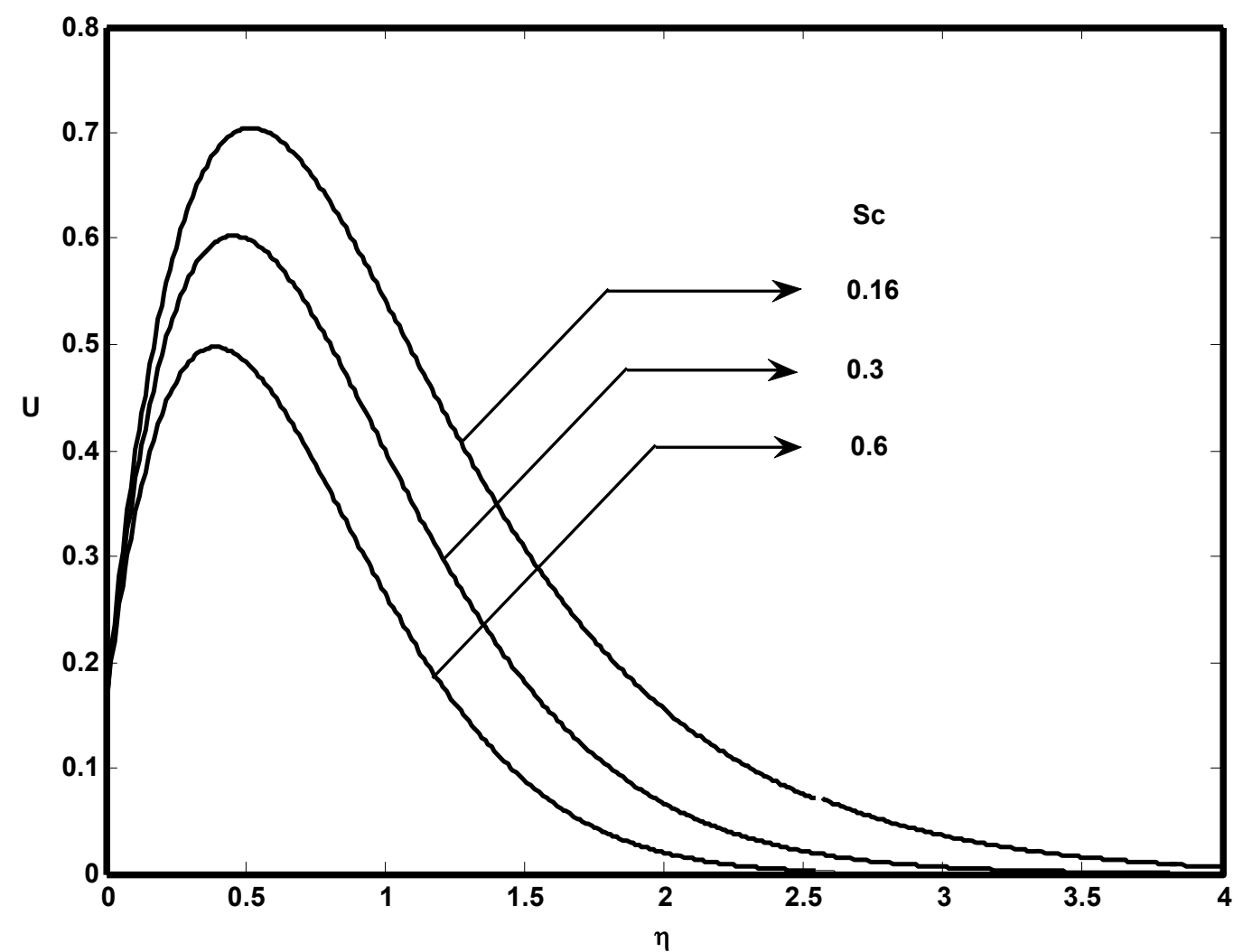

Fig.6. Velocity profiles for different values of Sc. 


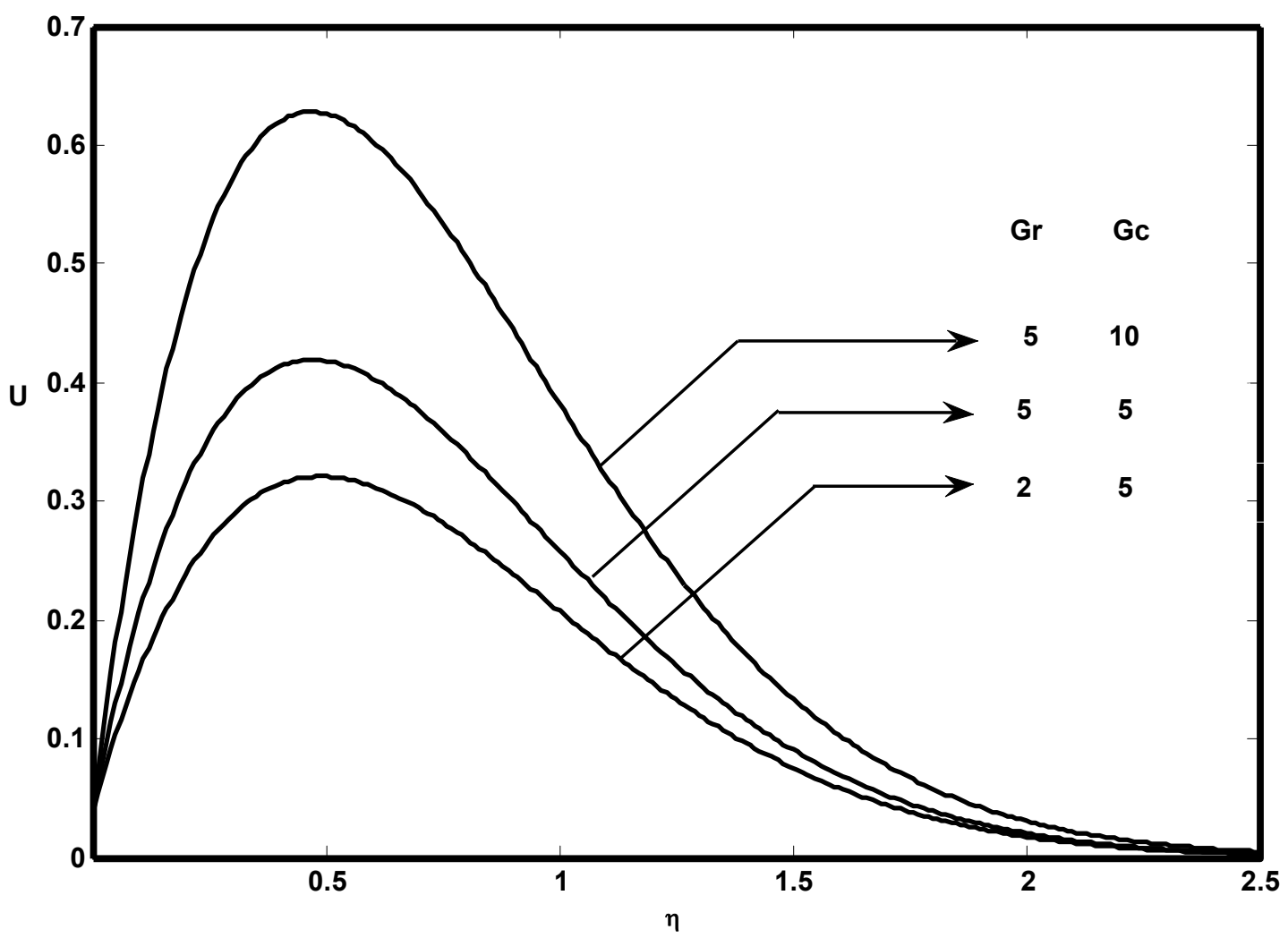

Fig.7. Velocity profiles for different values of Gr and Gc.

Figure 5 demonstrates the velocity profiles for different values of time $(t=0.2,0.4,0.6), K=2$, $\mathrm{Gr}=2, \mathrm{Gc}=5, R=4$ and $M=2$. It is observed that velocity increases with increasing values of time $t$.

Figure 6 illustrates the different values of the Schmidt number $(\mathrm{Sc}=0.16,0.3,0.6), K=2$ and $t=0.4$. The profiles have the common feature that the concentration decreases in a monotone fashion from the surface to a zero value far away in the free stream. It is observed that the concentration increases with the decreasing Schmidt number.

Figure 7 demonstrates the effects of different values of the thermal Grashof number $(\mathrm{Gr}=2,5)$, mass Grashof number $(\mathrm{Gc}=5,10), K=2$ and $\operatorname{Pr}=0.71$ on velocity at $t=0.2$. It is observed that velocity increases with increasing values of the thermal Grashof number or mass Grashof number.

\section{Concluding remarks}

An exact solution of MHD free convection flow with a parabolic starting motion of an infinite isothermal vertical plate in the presence of thermal radiation and chemical reaction The dimensionless governing equations are solved by the usual Laplace-transform technique. The effect of different parameters such as the thermal Grashof number, mass Grashof number, chemical reaction parameter, radiation parameter, magnetic field parameter and $t$ are studied graphically. The conclusions of the study are as follows:

(I) The temperature of the plate decreases with increasing values of the thermal radiation parameter.

(II) The concentration near the plate increases with decreasing values of the chemical reaction parameter or Schmidt number.

(III) Velocity increases with decreasing values of the magnetic field parameter or chemical reaction parameter or thermal radiation parameter. But the trend is just reversed with respect to time $t$. 


\section{Nomenclature}

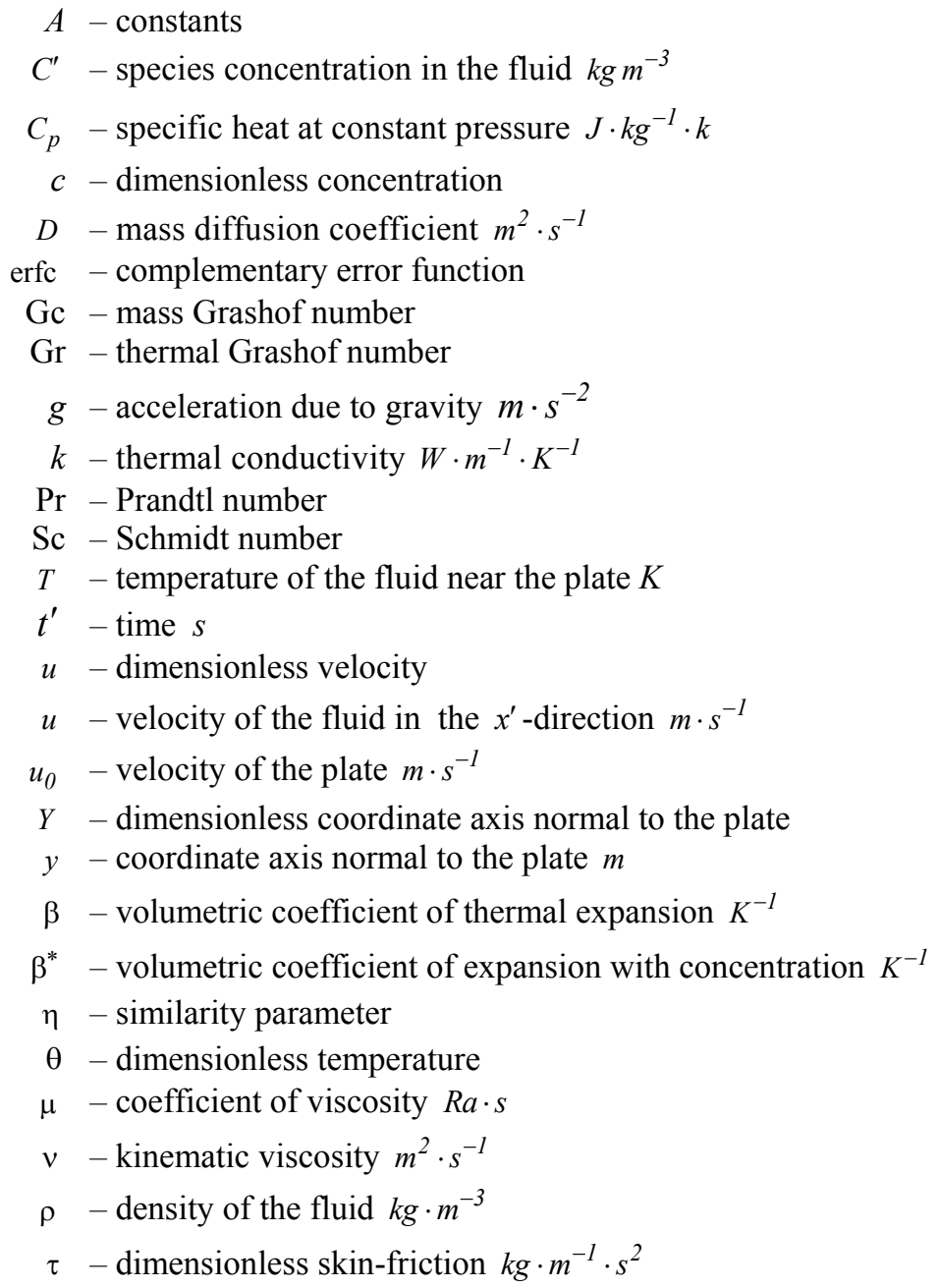

\section{Subscripts}

$w$ - conditions at the wall

$\infty$ - free stream conditions

\section{References}

[1] Chambre P.L. and Young J.D. (1958): On the diffusion of a chemically reactive species in a laminar boundary layer flow. - The Physics of Fluids, vol.1, pp.48-54.

[2] Das U.N., Deka R.K. and Soundalgekar V.M. (1994): Effects of mass transfer on flow past an impulsively started infinite vertical plate with constant heat flux and chemical reaction. - Forschung im Ingenieurwesen, vol.60, pp.284-287.

[3] Das U.N., Deka R.K. and Soundalgekar V.M. (1999): Effects of mass transfer on flow past an impulsively started infinite vertical plate with chemical reaction. - The Bulletin of GUMA, vol.5, pp.13-20.

[4] Muthucumaraswamy R. and Meenakshisundaram S. (2006): Theoretical study of chemical reaction effects on vertical oscillating plate with variable temperature, Theoret. - Journal of Applied Mechanics, vol.33, pp.245-257. 
[5] Gupta A.S., Pop I. and Soundalgekar V.M. (1979): Free convection effects on the flow past an accelerated vertical plate in an incompressible dissipative fluid. - Rev. Roum. Sci. Techn.-Mec. Apl., vol.24, pp.561-568.

[6] Kafousias N.G. and Raptis A.A. (1981): Mass transfer and free convection effects on the flow past an accelerated vertical infinite plate with variable suction or injection. - Rev. Roum. Sci. Tech. - Mec. Apl., vol.26, pp.11-22.

[7] Singh A.K. and Kumar N. (1984): Free convection flow past an exponentially accelerated vertical plate. Astrophysics and Space Science, vol.98, pp.245-258.

[8] Hossain M.A. and Shayo L.K. (1986): The skin friction in the unsteady free convection flow past an accelerated plate. - Astrophysics and Space Science, vol.125, pp.315-324.

[9] Jha B.K., Prasad R. and Rai S. (1991): Mass transfer effects on the flow past an exponentially accelerated vertical plate with constant heat flux. - Astrophysics and Space Science, vol.181, pp.125-134.

[10] Muthucumaraswamy R., Sathappan K.E. and Natarajan R. (2008): Mass transfer effects on exponentially accelerated isothermal vertical plate. - Int. J. of Appl. Math. and Mech., vol.4, No.6, pp.19-25.

[11] Soundalgekar V.M. and Takhar H.S. (1993): Radiation effects on free convection flow past a semi-infinite vertical plate. - Modeling, Measurement and Control, vol.B51, pp.31-40.

[12] Hossain M.A. and Takhar H.S. (1996): Radiation effects on mixed convection along a vertical plate with uniform surface temperature. - Heat and Mass Transfer, vol.31, pp.243-248.

[13] Das U.N, Deka R.K. and Soundalgekar V.M. (1996): Radiation effects on flow past an impulsively started vertical infinite plate. - J. Theo. Mech., vol.1, pp.111-115.

[14] Muthucumaraswamy R. and Janakiraman (2006): MHD and radiation effects on moving isothermal vertical plate with variable mass diffusion. - Theoret. Appl. Mech., vol.33, No.1, pp.17-29.

Received: March 21, 2014

Revised: September 10, 2015 